\title{
ESTUDO DO EFEITO DA PRESENÇA DE BOLHAS NA REMOÇÃO DE PARTÍCULAS SÓLIDAS EM UM MODELO FÍSICO DE DISTRIBUIDOR DE LINGOTAMENTO CONTÍNUO COM JATO ABERTO*
}

\section{Resumo}

\author{
Paula Rodrigues Caumo ${ }^{1}$ \\ Felipe das Dores Machado ${ }^{2}$ \\ Magda Beatriz Galant François ${ }^{3}$ \\ Antônio Cezar Faria Vilela ${ }^{4}$
}

A tecnologia da fabricação de aços, nas últimas décadas, apresentou grandes avanços, os quais resultaram em melhorias da qualidade, principalmente no que se refere à remoção de inclusões. As inclusões podem ser responsáveis por prejudicar as propriedades mecânicas por meio da geração de trincas ou fraturas entre outros defeitos. O distribuidor do lingotamento contínuo também pode atuar na remoção destas partículas. A eficácia desta função do distribuidor demanda o conhecimento das características do escoamento do aço líquido de modo que se possa propor modificações na configuração do distribuidor. Esse conhecimento pode ser obtido mediante a utilização de modelos físicos para simular os fenômenos envolvidos. $O$ objetivo deste trabalho é, a partir de um distribuidor com jato aberto, construído em acrílico e em escala 1:1, verificar o percentual de inclusões passantes pelo veio com ou sem a presença de bolhas. O trabalho foi executado com e sem tubo longo, sendo o tubo longo empregado para prevenir o arraste de ar para o interior do modelo. Os resultados foram obtidos por meio do ensaio de remoção de inclusões com o auxílio do ensaio de distribuição de tempos de residência. As variáveis utilizadas neste estudo são: presença bolhas e velocidade de lingotamento.

Palavras-chave: Bolhas; Modelagem física; Remoção de inclusões; Distribuidor.

\section{STUDY OF THE EFFECT OF THE PRESENCE OF BUBBLES IN THE REMOVAL OF SOLID PARTICLES IN A PHYSICAL MODEL OF TUNDISH OF CONTINUOUS CASTING WITH JET}

\begin{abstract}
The technology of the production of steel in the last decades has had great advance that has resulted in the improvement of the quality of steel, mainly for the removal of inclusions responsible for harming the mechanical properties of steels, generating cracks, fractures, etc. For this, the tundish of continuous casting has cooperated in the removal of inclusions. The effectiveness of this function of tundish demands the knowledge of the characteristics of the flow of the liquid steel so that they can propose modifications in the configuration of the tundish. This knowledge can be obtained through the use of a physical models to simulate the phenomena involved. The objective of this work is from a tundish with jet, built in acrylic and at the scale of 1:1, check the percentage of inclusions passing by came with or without the presence of bubbles. For the absence of bubbles will be placed a long tube, avoiding the presence of same. The results were obtained by means of the test of removal of inclusions with the aid of the test distribution of residence times.
\end{abstract}

Keywords: Bubbles; Tundish; Inclusion removal; Physical Model.

Graduanda de Eng. Metalúrgica, LaSid, DEMET, EE, UFRGS, Porto Alegre, RS, Brasil.

Eng. Químico, Mestre, LaSid, PPGE3M, EE, UFRGS, Porto Alegre, RS, Brasil.

Dr. INPL, Pesquisadora LaSid, UFRGS, Prof. ULBRA, Porto Alegre, RS, Brasil.

Prof. Dr.-Ing., LaSid, DEMET, PPGE3M, EE, UFRGS, Porto Alegre, RS, Brasil. 


\section{INTRODUÇÃO}

Com a crescente busca do setor industrial por aços com melhores características mecânicas, o processo de produção de aços tem seguido padrões necessários para que se possa obter um controle rígido quanto à quantidade, tamanho e morfologia de inclusões. Com essa preocupação, processos para melhorar a limpidez do aço estão sendo cada vez mais aprimorados. Algumas indústrias têm empregado a injeção de gás inerte no distribuidor de lingotamento contínuo, pois a mesma pode melhorar a flotação de inclusões, auxiliando na sua remoção. O borbulhamento de argônio diminui o número de inclusões no distribuidor, principalmente as macro-inclusões, o que é importante, pois elas podem atuar como concentradoras de tensões e, consequentemente, diminuir as propriedades mecânicas [1-2]. As inclusões podem ser originadas em diferentes etapas da aciaria, como por exemplo: a reoxidação do metal líquido por meio da exposição ao ar atmosférico, o processo de desoxidação e outros.

Convém ressaltar que o distribuidor é uma das últimas etapas do processo de lingotamento contínuo com volume e tempo de residência suficiente para as inclusões flotarem e serem retiradas do aço. Miki e Takeuchi [3] e Thomas, Yuan et al. [4] afirmam que para uma inclusão ser capturada por uma bolha, é essencial que a partícula entre no campo de interação da bolha, isto é, nas linhas de fluxos em torno da bolha as quais sobem uniformemente pelo aço líquido em direção à escória, auxiliando na sua remoção.

O estudo do escoamento interno do distribuidor e o fenômeno de remoção de inclusões são essenciais para que se consiga reduzir a quantidade de inclusões não metálicas no aço. Uma das alternativas utilizadas são os estudos via modelagem física do distribuidor, isto é a utilização de um distribuidor com jato aberto em acrílico em escala real (1:1) ou reduzida e que utiliza a água à temperatura ambiente como fluido simulador do aço líquido. $O$ objetivo deste trabalho é verificar o percentual de inclusões passantes pelo veio com ou sem a presença de bolhas. Para se obter a ausência de bolhas será colocado um tubo longo, evitando a presença das mesmas. Os resultados foram obtidos por meio do ensaio de remoção de inclusões com o auxílio do ensaio de distribuição de tempos de residência. As variáveis utilizadas neste estudo são: presença bolhas e velocidade de lingotamento.

\section{MATERIAIS E MÉTODOS}

Para a realização dos ensaios foi utilizado um modelo físico de distribuidor, em acrílico, pertencente ao Laboratório de Siderurgia da UFRGS. Este modelo é a reprodução de um equipamento de dois veios, instalado em uma usina siderúrgica para a fabricação de aços longos, (escala 1:1). Para que os resultados possam ser empregados é necessário que o escoamento e os fenômenos de flotação de inclusões sejam similares à situação real, desta forma o modelo foi construído utilizando os critérios de similaridade geométrica, cinemática e dinâmica [5]. A simulação física do aço líquido, utilizando a água à temperatura ambiente, é baseada na viscosidade cinemática da água a $25{ }^{\circ} \mathrm{C}$ que é similar à do aço na temperatura de processamento, aproximadamente $1600{ }^{\circ} \mathrm{C}$. Nesses ensaios foram testados dois tipos de configurações: presença ou não de bolhas, isto é, sem ou com tubo longo. No distribuidor (Figura 1) com 
jato aberto tem-se a presença de bolhas por consequência natural, geradas quando o jato atravessa a interface ar/água e para que se obtenha a ausência delas, é utilizado tubo longo, que por sua vez evita o arraste de bolhas. Também foi testado o efeito da variação da velocidade de lingotamento sobre a remoção de inclusões (1,8 m/min.; $2,2 \mathrm{~m} / \mathrm{min}$.; $2,6 \mathrm{~m} / \mathrm{min}$.). As medidas do distribuidor e tubo longo estão relatadas em Machado F. D. (2014) [6].

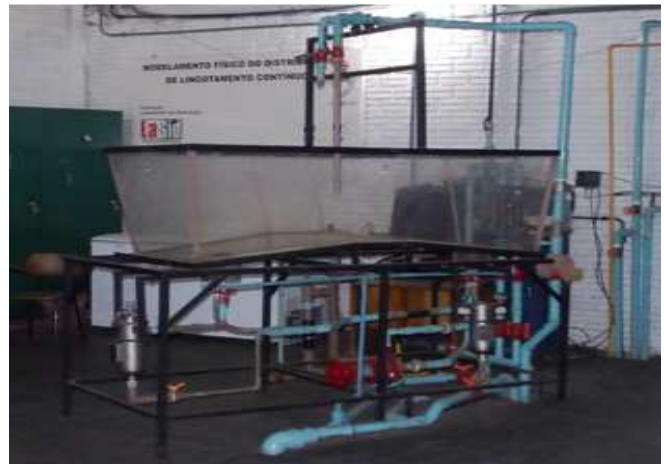

Figura 1. Modelo físico do distribuidor de lingotamento contínuo

\subsection{Ensaio de Remoção de Inclusões Com e sem a Presença de Bolhas}

O ensaio de remoção de inclusões, descrito em detalhes em Machado, (2014) [6], é executado com o distribuidor mantido em regime permanente. Após a estabilização do escoamento (10 min.) é realizada a injeção das partículas, que simulam inclusões de alumina, na forma de um pulso (3 segundos). Para formar uma solução possível de ser injetada no distribuidor, prepara-se a mistura de uma amostra de polietileno, (30 g), com uma solução de álcool etílico. O volume total da mistura é igual a $150 \mathrm{ml}$. Logo que a mistura é injetada, as micro partículas tendem a flotar em direção a superfície do distribuidor. Uma quantidade de partículas passa pelo distribuidor e se direciona para os veios de saída, devido ao arraste gerado pela velocidade do escoamento. Na saída do distribuidor existe uma peneira metálica adaptada no modelo, que captura as partículas passantes pelo veio permitindo a sua quantificação. Após dois tempos de residência teórica, as inclusões capturadas no filtro são recolhidas, secadas em estufa e pesadas. A configuração mais favorável para a remoção de inclusões é definida como aquela que permite 0 menor número de inclusões passantes pelos veios. O percentual de inclusões passantes é calculado por meio da Equação 1. Todos os ensaios são realizados em duplicata.

$\eta_{r}(\%)=\left(\frac{m_{c}}{m_{i}}\right) \times 100$

(1)

Onde $\eta_{\mathrm{r}}$ representa a eficiência de remoção, $m_{C}$, em gramas representa massa de partículas de simulação de inclusões coletadas na saída do distribuidor e $m_{i}$ em gramas representa a massa injetada no distribuidor.

\subsection{Ensaio de Distribuição de Tempos de Residência com e sem a Presença de Bolhas}

O ensaio de distribuição de tempos de residência, descrito em detalhes em Machado, (2014) [6], determina parâmetros como tempos mínimo e médio de 
residência além dos volumes característicos, e dá suporte aos resultados encontrados no ensaio de remoção de inclusões. $O$ método empregado para 0 ensaio de distribuição de tempos de residência consiste na injeção de um traçador ácido (ácido clorídrico com concentração de 6\%) e leitura da condutividade na saída do modelo ao longo do tempo de ensaio. O ensaio de DTR é realizado com o distribuidor mantido em regime permanente e a injeção do traçador é realizada após a estabilização do escoamento. O sensor de condutividade é posicionado em apenas um dos dois veios por motivo de simetria do distribuidor. A duração dos ensaios é de 30 minutos. Os dados capturados são tratados com o auxílio de uma planilha de cálculo que permite plotar as curvas DTRs e quantificar os resultados, como por exemplo, o tempo médio de residência $(\bar{\theta})$, o tempo mínimo, $\left(\overline{\theta_{m i n}}\right)$ e a variância adimensional $\left(\sigma_{\theta}^{2}\right)$, além dos volumes característicos. O equacionamento destas variáveis está descrito em Machado.

\section{RESULTADOS E DISCUSSÃO}

Nesta etapa, serão apresentados os resultados obtidos a partir dos ensaios realizados, avaliando o efeito da presença de bolhas na remoção de inclusões.

\subsection{Ensaio de Remoção de Inclusões com e sem a Presença de Bolhas}

$\mathrm{Na}$ Tabela 1 estão relatados os resultados encontrados no ensaio de remoção de inclusões. O percentual encontrado nas repetições 1 e 2 deve ser entendido como a quantidade de inclusões que entram no modelo e passam pelo veio.

Tabela 1.Resultados das porcentagens de inclusões passantes do ensaio de remoções de inclusões.

\begin{tabular}{ccccc} 
& & & \multicolumn{2}{c}{ Repetições } \\
\cline { 4 - 5 } Amostra & Velocidade & Tubo & $\mathbf{1}$ & $\mathbf{2}$ \\
\hline 1 & $1,8 \mathrm{~m} / \mathrm{min}$ & Não & $2,89 \%$ & $2,22 \%$ \\
\hline 2 & $2,2 \mathrm{~m} / \mathrm{min}$ & Não & $2,35 \%$ & $3,08 \%$ \\
\hline 3 & $2,6 \mathrm{~m} / \mathrm{min}$ & Não & $2,42 \%$ & $2,39 \%$ \\
\hline 4 & $1,8 \mathrm{~m} / \mathrm{min}$ & Sim & $22,48 \%$ & $22,67 \%$ \\
\hline 5 & $2,2 \mathrm{~m} / \mathrm{min}$ & $\operatorname{Sim}$ & $32,01 \%$ & $31,74 \%$ \\
\hline 6 & $2,6 \mathrm{~m} / \mathrm{min}$ & Sim & $33,93 \%$ & $35,74 \%$ \\
\hline
\end{tabular}

$\mathrm{Na}$ Tabela 1 é possível perceber que a presença de tubo longo aumenta o percentual de inclusões passantes pelo veio e que o aumento da velocidade também interfere na remoção de inclusões.

A Figura 2, e também a Tabela 1, mostram que a presença do tubo longo influencia fortemente no percentual de inclusões passantes pelo veio. Atribui-se este contraste a dois fenômenos presentes, ambos gerados pelo choque do jato de entrada com a interface ar/água. O primeiro deles é a quebra do vetor velocidade do escoamento gerada pelo choque do jato de entrada. O segundo fenômeno é o arraste de bolhas gerado quando o jato atravessa a interface ar/água (Figura 3). Neste último, as bolhas capturam as inclusões levando-as em direção a escória e reduzindo em porcentagem de inclusões passantes pelo 
veio [7]. A eficiência de captura e remoção das inclusões, entretanto, depende de vários fatores, tais como: tamanhos e distribuição espacial das bolhas; velocidade de lingotamento e grau de molhabilidade. [7]

A efetividade de remoção de inclusões pela presença de bolhas, além da distribuição de tamanhos, depende de relações angulares entre as bolhas e as partículas. As probabilidades de colisão e de aderência [8] das partículas também é afetada pelo fato que ambas estão em movimento. Convém ressaltar que partículas de dimensões menores são capturadas mais facilmente por bolhas de menores tamanhos. O grau de molhabilidade da partícula inclusionária pelo aço líquido afeta substancialmente a tendência da partícula em ser aderida e arrastada pela bolha. Partículas molháveis pelo aço líquido tendem naturalmente a permanecer dispersas no aço e as inclusões nãometálicas (com composição predominante de compostos de alumina e sílica) não são molháveis pelo aço líquido, e podem ser capturadas pelo trem de bolhas, e facilmente flotadas [8].

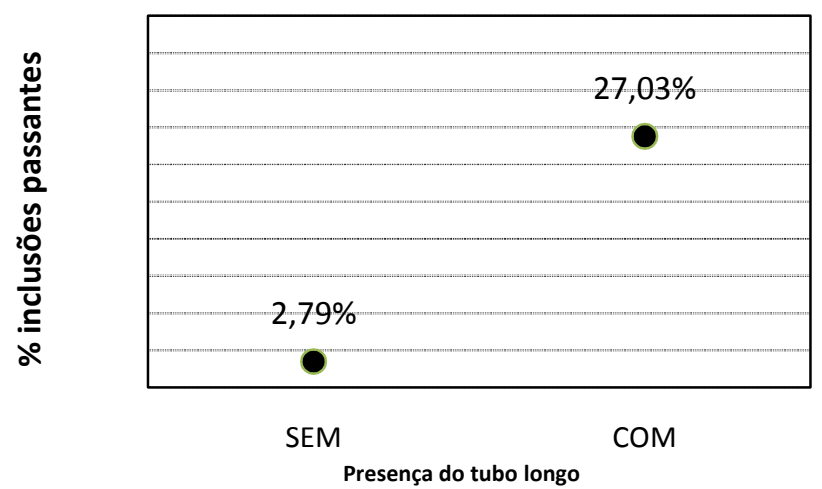

Figura 2. Porcentual de inclusões passantes em função da presença de tubo longo

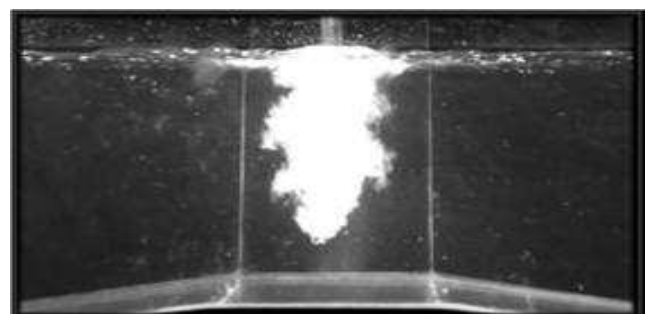

Figura 3. Arraste de bolhas para o seio do banho metálico gerado na ausência do tubo longo

Nos ensaios sem tubo longo é importante ressaltar que a ausência deste pode aumentar a formação de inclusões via reoxidação do banho metálico. Logo, deve-se lembrar estes ensaios não consideram inclusões geradas pela reoxidação via exposição do aço líquido à atmosfera. A quantificação deste fenômeno deve alterar os percentuais de inclusões passantes nesses ensaios. $\mathrm{Na}$ indústria, para obter a presença de bolhas injeta-se argônio, de forma a auxiliar na flotação de inclusões e ao mesmo tempo evitar a reoxidação do banho metálico.

$\mathrm{Na}$ Figura 4 tem-se os resultados de inclusões passantes em três velocidades de lingotamento. Observa-se que 0 aumento da velocidade afeta principalmente as configurações com tubo longo, dado que há um maior aumento no porcentual de inclusões passantes de 20,55 para $32,18 \%$ (com tubo longo). Já a configuração sem tubo longo não apresentou grande 
diferença nos resultados quando a velocidade aumenta (2,68 para 2,73\%). Essa diferença de resultados é o efeito da presença de bolhas na configuração sem tubo longo, no qual elas colaboram em diminuir o efeito da velocidade de lingotamento sobre as inclusões passantes pelo veio.

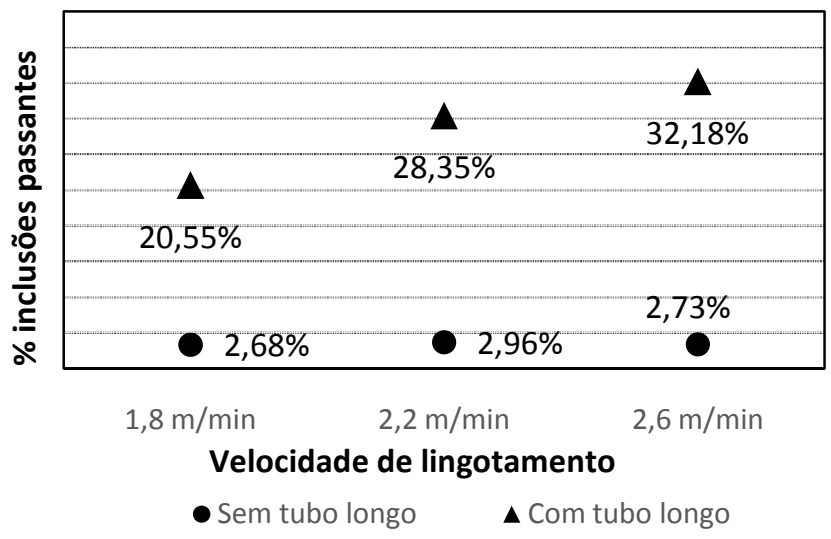

Figura 4. Porcentual de inclusões passantes em função da combinação entre a presença do tubo longo e a velocidade de lingotamento.

\subsection{Ensaios de Distribuição de Tempos de Residência}

Os resultados do ensaio de distribuição de tempos de residência (DTR) são apresentados a seguir nas configurações: presença de bolhas (sem tubo longo) e ausência de bolhas (tubo longo)e em três velocidades de lingotamento. Devido à velocidade não apresentar efeito sobre as curvas de DTR, todos os resultados do ensaio de distribuição de tempos de residência (tempo médio de residência, tempo mínimo de residência e frações de volumes característicos) são apresentados com os resultados das médias dos valores para diferentes velocidades, além do resultado para cada velocidade.

\subsubsection{Tempos médio e mínimo de residência}

O tempo de residência de um fluido em um reator é definido como o tempo que cada elemento de fluido dispende no percurso do reator [9]. No ensaio de DTR o tempo médio de residência indica que quanto maior for esse valor melhor será a remoção das partículas inclusionárias. A Figura 5 apresenta o tempo médio de residência normalizado para as configurações testadas neste trabalho. É possível verificar que a melhor configuração é a configuração com tubo longo, cuja média dos resultados para as velocidades de lingotamento apresentada em azul é igual a 0,550. A configuração sem tubo longo obteve média igual a 0,520, logo teve um menor tempo para a flotação das inclusões. 


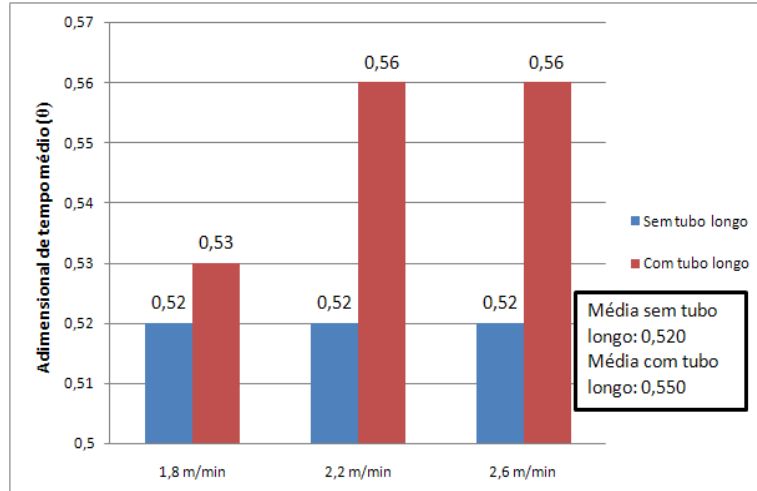

$(\bar{\theta})$

Figura 5. Adimensional de tempo médio de residência

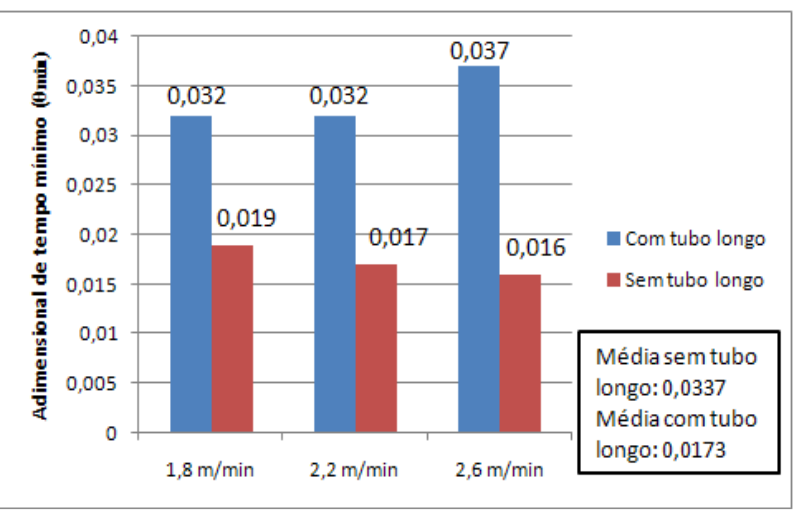

Figura 6. Tempo mínimo de residência $\left(\theta_{\min }\right)$

O tempo mínimo de residência indica o tempo mínimo que um fluido dispende no percurso do reator. Quanto maior o tempo mínimo melhor as possibilidades de flotação de inclusões. Os resultados de tempo mínimo de residência normalizado são apresentados na Figura 6. A configuração com tubo longo apresenta o pior resultado $(0,0173)$. Esse resultado deve-se ao escoamento preferencialmente descendente e com curto-circuito (Figura 9). A configuração que apresenta maior tempo mínimo e, consequentemente, maior remoção de inclusões é a configuração sem tubo longo cujo valor médio do adimensional de tempo mínimo é igual a 0,0337.

\subsubsection{Frações de volumes característicos}

O volume pistonado representa a região de movimento lamelar sem mistura [9] e está associado a melhores condições de flotação de inclusões. Com relação à fração de volume pistonado, Sahai e Ahuja, 1986, discutem a forma da curva DTR para o estabelecimento de uma equação. Os autores consideram que se na curva DTR o tempo de concentração mínima $\left(\theta_{\min }\right)$ for diferente do tempo de concentração máxima, $\left(\theta_{\max }\right)$, então existe dispersão axial e a fração de volume em escoamento pistonado seria calculada por meio da equação 2 [9]:

$V_{p d}=\left(\frac{\theta_{\min }+\theta_{\max }}{2}\right)$

Logo, quanto maior for esse valor, melhor é a condição de remoção de inclusões no distribuidor. A Figura 7 mostra que o maior valor de volume pistonado disperso para as configurações estudadas é atingido com a utilização do tubo longo $(11,5 \%)$. Enquanto que o menor valor é para a configuração sem tubo longo $(9,1 \%)$.

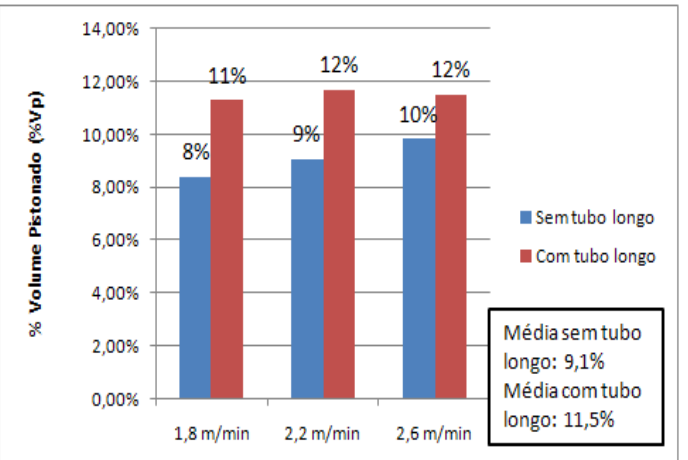

Figura 7. Fração de volume pistonado disperso $(\mathrm{Vpd})$

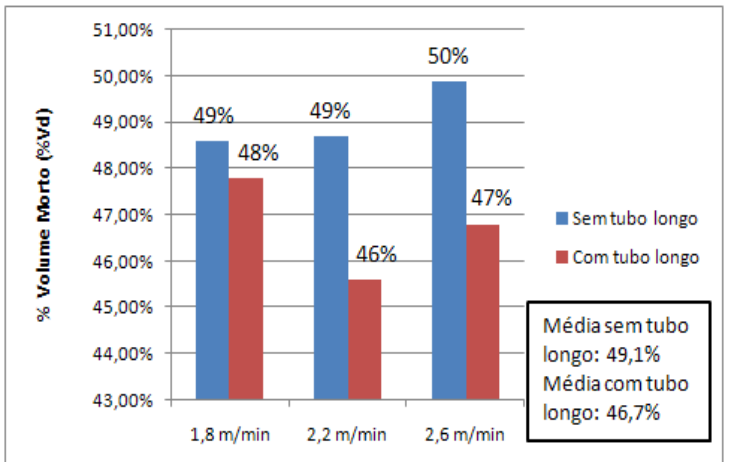

Figura 8. Fração de volume morto (Vd) 
volume morto representa a porção de líquido que se move tão lentamente que pode ser assumida como estagnada [9]. Para uma eficiente remoção de inclusões espera-se que o volume morto seja o menor possível. A condição de menor volume morto (Figura 8) é a configuração com tubo longo (46,7\%). A pior condição de volume morto é a configuração sem tubo longo $(49,1 \%)$.

O curto-circuito no distribuidor é prejudicial devido à diminuição das possibilidades de flotação das inclusões, pois o escoamento se direciona diretamente para os veios. Como apresentado na Figura 9, a configuração sem tubo longo apresentou menor curto circuito $(3,4 \%)$, enquanto que a configuração com tubo longo apresentou $(11,1 \%)$.

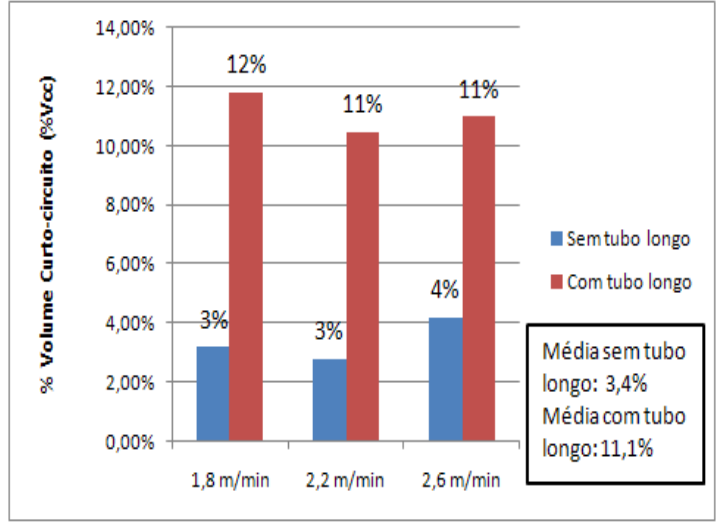

Figura 9. Fração de volume de curto-circuito (Vcc).

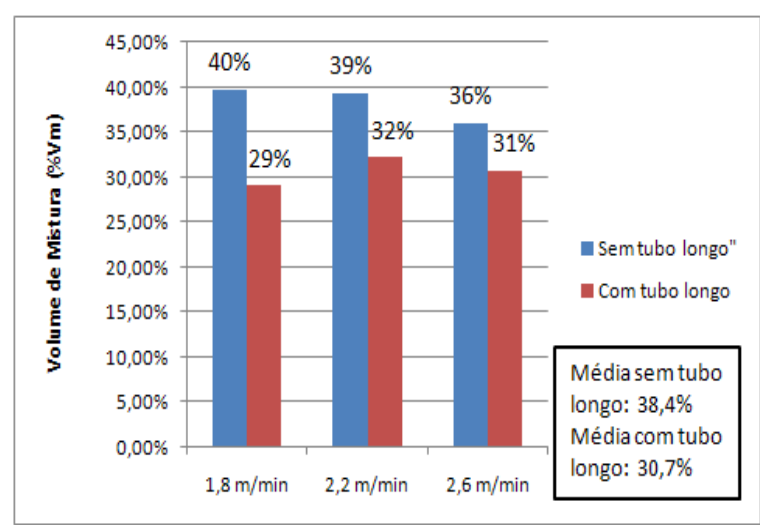

Figura 10. Fração de volume de mistura $(\mathrm{Vm})$.

A Figura 10 mostra o comparativo das frações de volume de mistura para as diferentes configurações e o maior valor encontrado é para a configuração sem tubo longo $(38,4 \%)$. Atribui-se a isso pela presença de bolhas, pois as mesmas auxiliam no volume de mistura pela turbulência gerada.

\subsubsection{Relação entre volume pistonado disperso e volume morto}

A relação entre o volume pistonado disperso $(V p d)$ e o volume morto $(V d)$ é um bom índice para o estudo da remoção de inclusões a partir do ensaio de distribuição de tempo de residência. Como o volume pistonado disperso auxilia no direcionamento das inclusões à escória em escoamentos preferencialmente ascendentes e o volume morto diminui o tempo de residência do aço (prejudicial à flotação das inclusões) espera-se que o primeiro aumente 0 máximo possível e o último diminua. Logo, quanto maior for a relação $\mathrm{Vpd} / \mathrm{Vd}$, melhor são as condições de flotação de inclusões.

$\mathrm{Na}$ Figura 11 a maior relação $\mathrm{Vpd} / \mathrm{Vd}$ é encontrada na configuração com tubo longo $(0,250)$ caracterizando o melhor escoamento para flotação de inclusões. O menor resultado de 0,187 pertence à configuração sem tubo longo. 


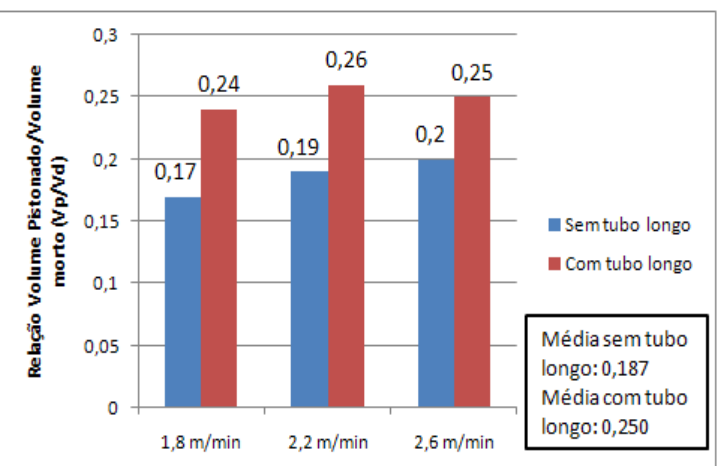

Figura 11. Relação entre volume pistonado disperso e volume morto (Vpd/Vd) para as configurações estudadas.

Sob o ponto de vista do ensaio de distribuição de tempo de residência, a configuração com tubo longo é a melhor dentre as estudadas neste trabalho. Os motivos são listados a seguir:

- Maior tempo médio de residência;

- Maior volume pistonado disperso;

- Menor volume morto;

- Maior relação $\mathrm{Vpd} / \mathrm{Vd}$.

O distribuidor em questão foi construído com base em uma planta real existente para aços longos. O modelo para a realização dos ensaios foi escolhido pelos critérios: menor tamanho e menos complexidade. Dado que o distribuidor para aços longos além de ser maior, também exige uma complexidade de manuseio superior, pois seu processo de fabricação demanda mais atenção quanto à limpidez do aço.

Comparando os resultados dos ensaios de remoção de inclusões com os resultados de distribuição de tempos de residência percebe-se que os resultados obtidos nos ensaios de tempo de residência não foram confirmados por meio dos resultados dos ensaios de remoção de inclusões. Isto se deve, prioritariamente ao fato que as inclusões, auxiliadas pelas bolhas, apresentam um comportamento diferente do escoamento, com uma velocidade de ascensão maior.

\section{CONCLUSÕES}

Conclui-se, a partir do ensaio de remoção de inclusões, que a presença de bolhas têm efeitos positivos para a remoção de inclusões no distribuidor de lingotamento contínuo com jato aberto. Com a utilização de tubo longo o percentual de inclusões passantes foi de $27,03 \%$, utilizando a configuração sem tubo longo onde há 0 arraste de bolhas o percentual de inclusões passantes foi de $2,79 \%$.

Conclui-se a partir do ensaio de distribuição de tempos de residência que a configuração com tubo longo apresentou melhores resultados em relação ao escoamento no interior do distribuidor de lingotamento contínuo. Avaliando os resultados obtidos nos ensaios é evidente que mesmo uma configuração que possui a maioria dos resultados de escoamento favoráveis para remoção de inclusões, esta configuração pode não ser a melhor opção. Nesse caso, isso pode ter ocorrido devido à ausência do arraste de bolhas para o interior do distribuidor, aumentando o percentual de inclusões passantes pelo veio. 
Conclui-se que o aumento na velocidade de lingotamento utilizando a configuração com tubo longo provoca um aumento no percentual de inclusões passantes de 20,55 para $32,18 \%$ e a configuração sem tubo longo não apresenta grandes acréscimos no percentual de inclusões passantes, de 2,68 para $2,73 \%$. Essa discrepância de resultados é o efeito da presença de bolhas na configuração sem tubo longo, no qual elas colaboram em diminuir o efeito da velocidade de lingotamento sobre as inclusões passantes pelo veio.

\section{Agradecimentos}

A Fundação Luiz Englert pela bolsa de iniciação científica concedida.

\section{REFERÊNCIAS}

1 Rogler, J. P; Heaslip, L. J \& Mehrvar, M - Inclusion Removal in a Tundish by Gas Bubbling. Canadian Metallurgical Quartely. Vol. 43, No. 3, 2004, p. 407-416.

2 Marique, C; Dony, A \& Nyessen, P - The Bubbling of Inert Gas in the Tundish: A Means to Improve the Steel Cleanliness. In Tundish Metallurgy-Volume II. ISSC; USA, 1991, p. 49-55.

3 Miki, Y \& Takeuchi, s - Internal Defects of Conitnuous Casting Slabs CausaebyAssimetric Unbalanced Steel Flow in Molde. ISIJ International, vol. 43, no. 10,2003, p. 1548-1555.

4 B.G. Thomas, Q. Yuan, L. Zhang, And S.P. Vanka - Flow Dynamics and Inclusion Transport in Continuous Casting of Steel 2003 NSF Design, Service, and Manufacturing Grantees and Research Conf. Proceedings, R.G. Reddy, ed (Burmingham, AL, Jan. 6-9 2003), University of Alabama, Tuscaloosa, AL 35498,USA, p. 2328-2362.

5 François, B. M. G., Machado, D. F. E Vilela, A. C. F. Remoção de inclusões em modelos físicos de distribuidores de lingotamento contínuo de aços: uma revisão bibliográfica. XXXXIV Seminário de aciaria, Associação Brasileira de Metalurgia e Materiais - ABM, Araxá, MG, Brasil, 2013.

6 Machado, F. D. Modelagem física de remoção de inclusões em distribuidor de lingotamento contínuo de tarugos. Dissertação de mestrado a ser defendida em 24 de março de 2014. Porto Alegre: Universidade Federal do Rio Grande do Sul, 2014.

7 Rogler, J. P.; Heaslip, L. J.; Mehrvar, M. Inclusion removal in a tundish by gas bubbling. Canadian Metallurgical Quarterly, v. 43, p. 407-16, 2004.

8 Yokoya, S, Takagi, S; Iguchi, M; Marukawa, K \& Hara, S - Formation of Fine Bubble through Swirling Motion of Liquid Metal in the Metallurgical Container ISIJ International, Vol. 40 (2000), No. 6, p. 572-577.

9 François, M. B. G.; Bock, M. L. ; Mattiello, M. A. Z.; Vilela, A. C. F. Contribuição ao entendimento das curvas de determinação de tempos característicos(DTR) e dos volumes característicos em modelos físicos de distribuidor de lingotamento contínuo. 15ํㅡㄹ Conferencia de Aceria, Instituto Argentino de Siderurgia - IAS, San Nicolás/Argentina, 2005. 